\title{
Study on Effect of Different Porosity on Thermally Driven Heat Transfer in a Centrifugal Force Field
}

\author{
Xia Jie ${ }^{1, a^{\star}}$, Chang Hai-ping ${ }^{2, b}$, and Tang Hou-xiang ${ }^{3, c}$ \\ ${ }^{1}$ Department of Theory Training, Flight Drillmaster Training Base, China \\ ${ }^{2}$ College of Energy and Power Engineering, Nanjing University of Aeronautics and Astronautics, China \\ ${ }^{3}$ Department of Basic Course, Automobile Management Institute, China \\ xjxj china@163.com, ${ }^{\mathrm{b}}$ chppe@nuaa.edu.cn, ${ }^{\mathrm{C}}$ tanghouxiang@163.com
}

Key words: porous medium; porosity; thermally driven

Abstract: Based on the exploiture of turbine blade super cooling technology, porous medium is installed in a new kind of cooling configuration with cooling tunnels. Experiments and numerical simulations are carried out to investigate the thermally driven heat transfer rules in the new kind of cooling configuration with different porosity in a centrifugal force field. The results of experiments and numerical simulations are consistent basically. The results of study show that the heat transfer rules of the new cooling configuration are identical under different porosity. The thermally driven heat transfer of the cooling configuration filled with porous medium can be enhanced with increase of the rotate speed, heat flux and cooling air speed. At the same time, the heat transfer effect can be weakened with increase of the porosity under high porosity.

\section{Introduction}

That if increasing gas's inlet temperature effectively and reliably is the root of enhancing thermal efficiency and thrust-weight ratio according to gas turbine's thermal circulation. Turbine blade's cooling design will be the key to solve question to reach future high-powered engine's technology index. Focusing on the situation, Professor Chang Hai-ping and Academician Guo Zeng-yuan brought up a new cooling technique based on the theory of thermal drive [1]. Slight cooling channels are the core of the technique. Cooling medium is put into the slight cooling channels. Porous medium is installed in channels to enhance slight channels' structure intensity. At the same time, flowing of the fluid in the porous medium exist dispersion effect. It can make molecular groups mixed radially, make radial temperature distribution uniform and even, and eventually enhance heat exchange evidently [2-4]. Along with enhancing heat exchange, resistance to flow also augments a lot. But the increasing resistance can be overcome with enough buoyancy. Based on the fundamental study[5-7], in this paper, experiments and numerical simulations were carried out to investigate the thermally driven heat transfer rules in the new kind of cooling configuration with different porosity and compare their heat transfer effect.

\section{Experimental Research}

\section{Experimental Facility}

Fig1 is a scheme of experimental apparatus. The whole experimental apparatus contains power system, heating system, cooling system and measuring system, etc. Cooling air enters hollow shaft, passes gas collection case into experimental model, and streams out of the turntable. There are two layers hole boards in the gas collection case to make gas enter the experimental model equably to simulate the turbine blade's work state.

Fig2 and Fig3 are photos and sketch map of the testpieces. In order to obtain the closed channels illustrated in Fig3, one body shell, five inner hulls and two end plates were manufactured. In the closed channels, foamed metals made with colomony are used in the porous medium area(porosity is $98 \%$ and $93 \%$ ), 
which is illustrated concretely in Fig2. The external dimension of the testpieces is $50 \mathrm{~mm} * 36 \mathrm{~mm} * 38 \mathrm{~mm}$. Except that the width of the porous medium channel is $3 \mathrm{~mm}$, the width of all the others is $1 \mathrm{~mm}$.

Contract grant sponsors: National Natural Science Foundation of China(50276027) and the Foundational Research of the National Defence (J1400D001)

In the experiments, the heating theca posted on the body shell heats the cycle channels, and adjusting electric current can create different heat. At the same time, cooling air goes through the inner hulls to form cooling channels, and takes away the heat discharged by cycle fluid. Adjusting the air flux can create different cooling air speed. The operational range of the experiments includes: heat flux $\left(5390-48500 \mathrm{w} / \mathrm{m}^{2}\right)$, cooling air speed $(32.1-96.2 \mathrm{~m} / \mathrm{s})$ and rotate speed (300-1380 $/ \mathrm{min})$.

In order to research the thermo-driving heat transfer effectiveness of fluid in the closed channels installed with porous medium, the author defines a parameter $(\mathrm{KH})$ of capacity of thermo-driving. It characterizes the capacity of the fluid in the closedchannels, which takes heat to the cooling end with the circular flow of thermo-driving. The concrete definition just as follows:

$$
K H=\frac{q}{T_{\max }-T_{\min }}
$$

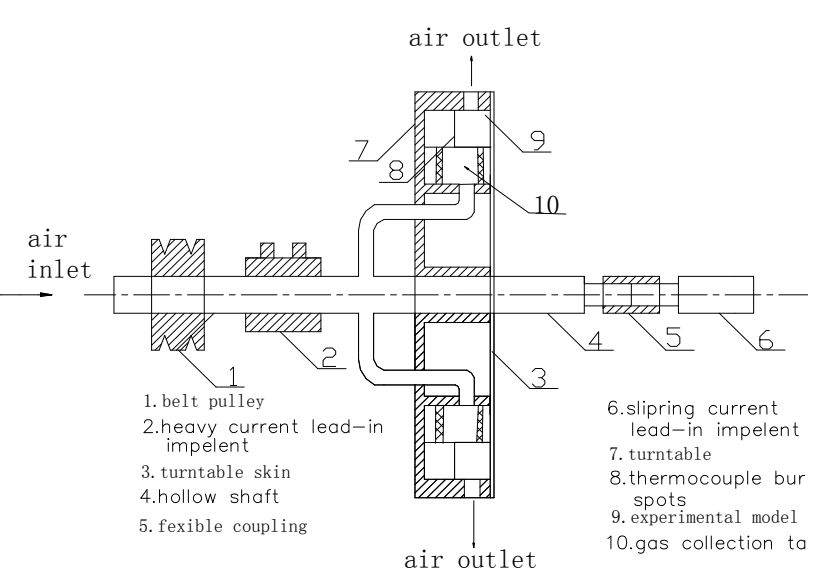

Fig.1 Schematic of experimental apparatus

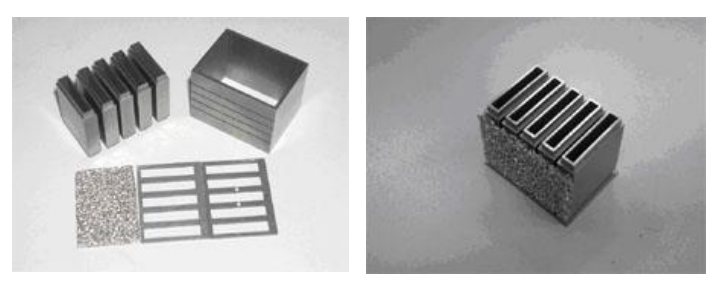

Where, $q$ is heat flux of the heating surface, $T_{\max }$ and $T_{\min }$ respectively is the highest and lowest temperature measured from the surface of the walls along with the orientation of the circular flow of thermo-driving.

\section{Experimental Results and Discussion}

Fig4, Fig5 and Fig6 illustrate the diagrams that the temperature on the heating surface changes along with heat flux, rotating speed and intake velocity of the cooling air, here the porosity is $93 \%$ in porous area. As illustrated in Fig4, with the same heat flux density, the temperature of the heating end along with the positive direction of the $\mathrm{Y}$ axis consistently falls, because after absorbing heat, the temperature of fluid increases and the density decreases, and the fluid affected by centrifugal force moves along with the negative direction of the $\mathrm{Y}$ axis. That demonstrates the flow of thermo-driving takes place in the enclosure after being filled with porous medium (If there is no thermo-driving but only
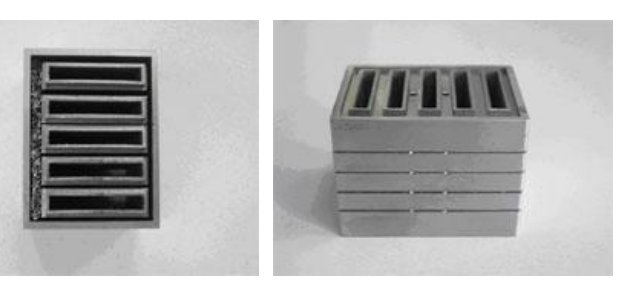

Fig.2 Photos of experimental mode

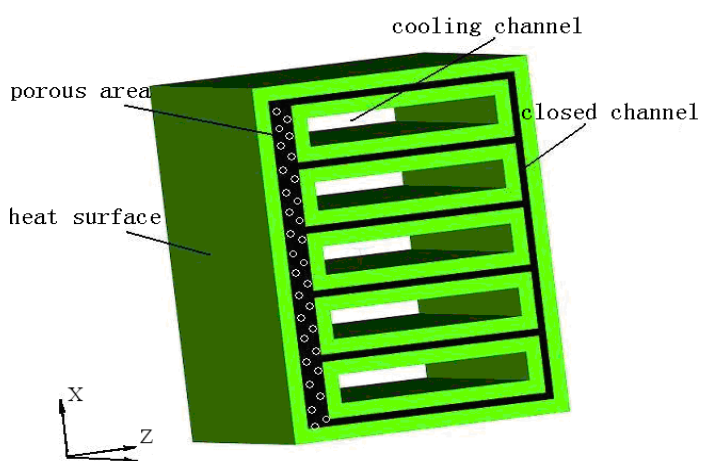

Fig.3 Schematic of experimental model thermo-conduction, the temperature on the heating surface will be uniformity, and along with the Y axis will have no change. The temperature of the heating end along with the positive direction of the $Y$ axis consistently increases under rotation. That is contrary to the situation under no rotation.). Furthermore, the temperature at all the measuring points increase in varying degrees along with increase of heat flux. The higher the heat flux, the greater the temperature difference between the highest and lowest measuring points. That demonstrates heat flux increases and thermo-driving capacity improves and then thermo-driving heat transfer effectiveness continuously rises. Fig5 and Fig6 illustrate the temperature at all the measuring points decrease and the temperature difference between the highest and lowest measuring points decreases. That demonstrates 
rotating speed (centrifugal acceleration) or intake velocity of the cooling air increases, thermo-driving heat transfer effectiveness also rises.

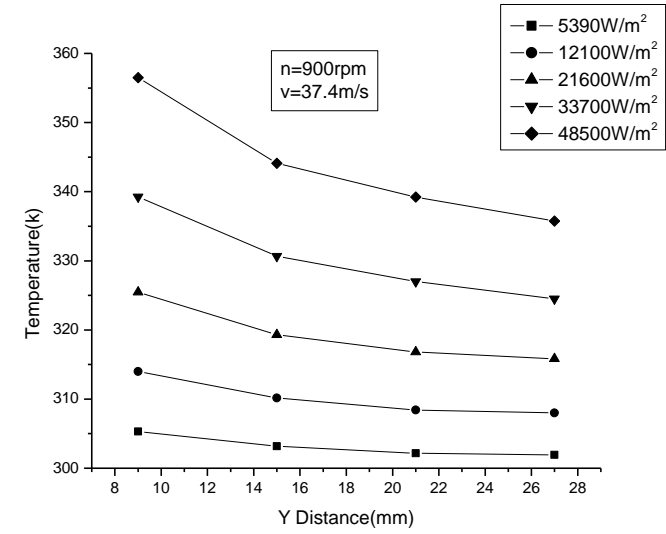

Fig.4 Curves of temperature varying with heat fluxes

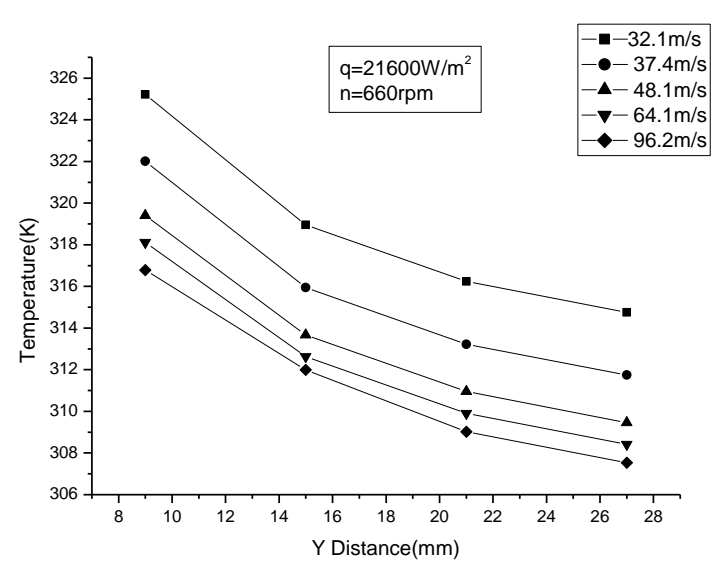

Fig. 6 Curves of temperature varying with cooling air speeds

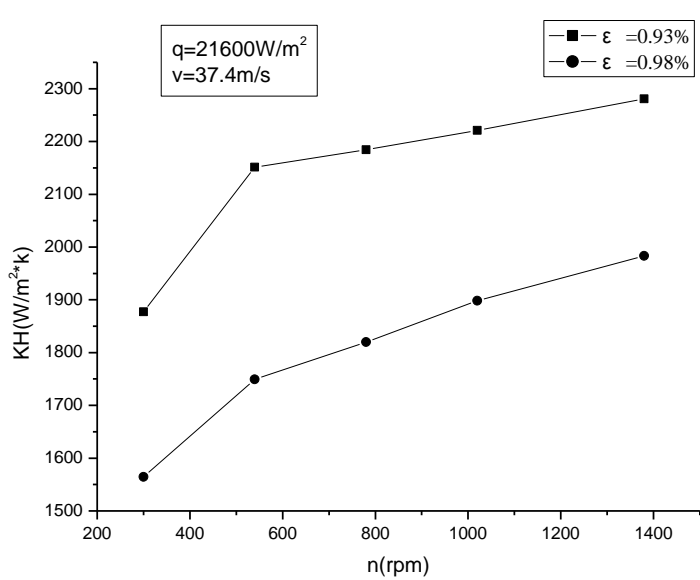

Fig.8 Curves of KH varying with rotating speeds under two kinds of porosity

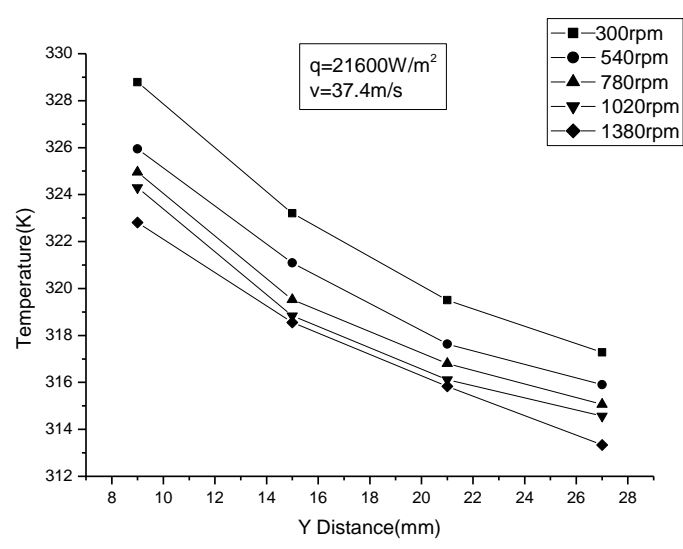

Fig.5 Curves of temperature varying with rotating speeds

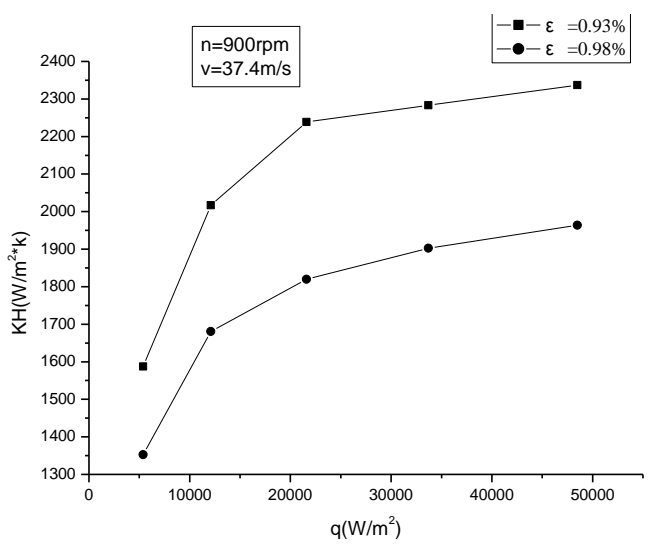

Fig.7 Curves of KH varying with heat fluxes under two kinds of porosity

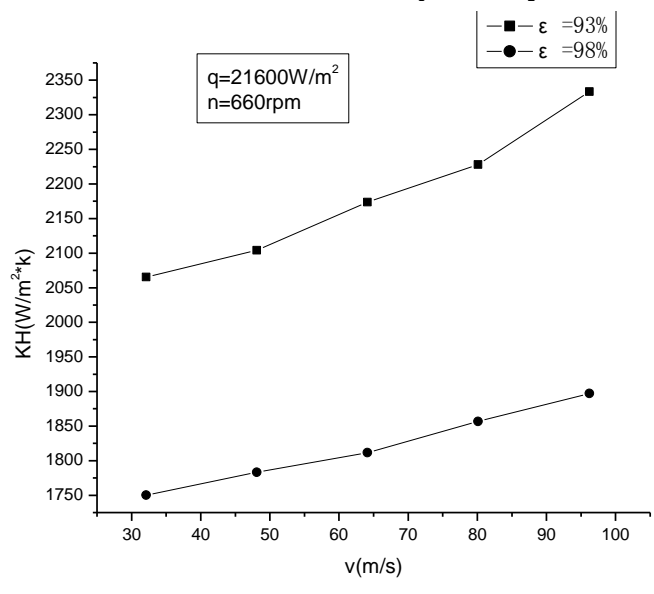

Fig.9 Curves of KH varying with cooling air speeds under two kinds of porosity

The paper researches the thermo-driving heat transfer in the enclosure with porous medium whose porosity is $93 \%$, meanwhile, it makes a comparison between the results and those porosity is $98 \%$. The 
research demonstrates the enclosure has the same temperature distribution rule under two kinds of porosity. So, the temperature distribution chart when the porosity is $98 \%$ is not given in this paper. Fig7, Fig8 and Fig9 respectively demonstrate the diagrams of the comparison of the parameters of thermo-driving heat transfer capacity between two kinds of porosity when heat flux, rotating speed and cooling air speed change. The figures illustrate that the parameter of thermo-driving heat transfer capacity increases and thermo-driving capacity improves along with increase of heat flux, rotating speed and cooling air speed under two kinds of porosity. Although the heat transfer rule is the same, the enclosure with $93 \%$ porosity has much better thermo-driving heat transfer effectiveness than the other. The reason is as follows. In porous medium area, solid medium's heat conduction coefficient is higher than the fluid in this examination. So, solid medium's heat conduction and convection heat transfer between solid medium and fluid have very important action in porous medium area. The contact area between solid medium and fluid increases along with decrease of porosity. Consequently, it strengthens convection heat transfer in porous medium area and improves the enclosure's thermo-driving heat transfer capacity and effectiveness.

\section{Numerical Research}

\section{Mathematical Model}

In order to find out the fluid's flow and heat transfer character, flow and temperature field in the enclosure with porous medium and the fluid's thermo-driving heat transfer capacity under different porosity, the paper carries out numerical simulation. The paper sets up mathematical model according to experimental model's size in numerical simulation. Basic equations are as follows.

no porous medium

$$
\begin{aligned}
& \frac{\partial\left(\rho u_{i}\right)}{\partial x_{i}}=0 \\
& \frac{\partial\left(\rho u_{i} u_{j}\right)}{\partial x_{j}}=-\frac{\partial P}{\partial x_{i}}+\frac{\partial \tau_{i j}}{\partial x_{j}}+\rho g_{i}-2 \rho e_{i j k} \omega e_{j} u_{k}+\rho \frac{\partial}{\partial x_{i}}\left(\frac{1}{2} \omega^{2} R^{2}\right) \\
& \frac{\partial\left[u_{i}(\rho E+P)\right]}{\partial x_{i}}=\frac{\partial}{\partial x_{i}}\left[k \frac{\partial T}{\partial x_{i}}+u_{j}\left(\tau_{i j}\right)\right]
\end{aligned}
$$

with porous medium

$$
\begin{aligned}
& \frac{\partial\left(\rho u_{i}\right)}{\partial x_{i}}=0 \\
& \frac{1}{\varepsilon^{2}} \frac{\partial\left(\rho u_{i} u_{j}\right)}{\partial x_{j}}=-\frac{\partial P}{\partial x_{i}}+\frac{\partial \tau_{i j}}{\partial x_{j}}-\frac{\mu}{\alpha} u_{i}+\rho g_{i}-2 \frac{\rho}{\varepsilon} e_{i j k} \omega e_{j} u_{k}+\rho \frac{\partial}{\partial x_{i}}\left(\frac{1}{2} \omega^{2} R^{2}\right) \\
& \frac{\partial\left[u_{i}(\rho E+P)\right]}{\partial x_{i}}=\frac{\partial}{\partial x_{i}}\left[k_{e f f} \frac{\partial T}{\partial x_{i}}+u_{j}\left(\tau_{i j}\right)_{e f f}\right]
\end{aligned}
$$

$\omega$ is gyral angle velocity; $\varepsilon$ is porosity; $k_{\text {eff }}$ is effective heat conduction coefficient $\left(k_{e f f}=\varepsilon k_{f}+(1-\varepsilon) k_{s}, k_{f}\right.$ is fluid heat conduction coefficient, $\mathrm{k}_{\mathrm{s}}$ is solid heat conduction coefficient); $\mathrm{E}$ is fluid inner enerry; $\alpha$ is the infiltration rate of porous medium.

The boundary conditions according to experimental parameters are as follows: Heating surface is supposed changeless heat flux; cooling surface carries through heat transfer with liquid in the enclosure and cooling air, so it is supposed coupling surface; the inlet and outlet surface of the cooling air channel are supposed velocity inlet and pressure outlet; all the other surfaces are supposed as perfect adiabatic walls.

In the numerical simulation we adopt Gambit to bulid grid and Fluent's Coupled Implicit Steady Solver.

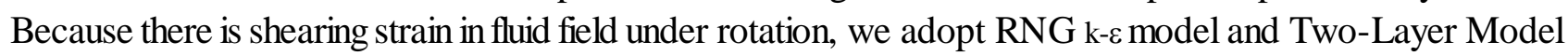
near wall. 


\section{Numerical Results and Analysis}

Fig10 is temperature distributing of the experimental model. The temperature on the heating surface along with the positive direction of the $\mathrm{Y}$ axis falls, which is consistent with experimental result. In order to verify numerical result, the paper compares numerical heating surface temperature with experimental result. Fig11 is the comparison of numerical with experimental result under the same conditions $\left(\mathrm{q}=5390 \mathrm{~W} / \mathrm{m}^{2}, \mathrm{n}=900 \mathrm{rpm}\right.$, $\mathrm{v}=37.4 \mathrm{~m} / \mathrm{s}$ ). Fig1 1 illustrates that numerical and experimental result's change trand is the same. Numerical result is five degrees higher than experimental result, because the absolute adiabatic condition is not realized in experimentation.
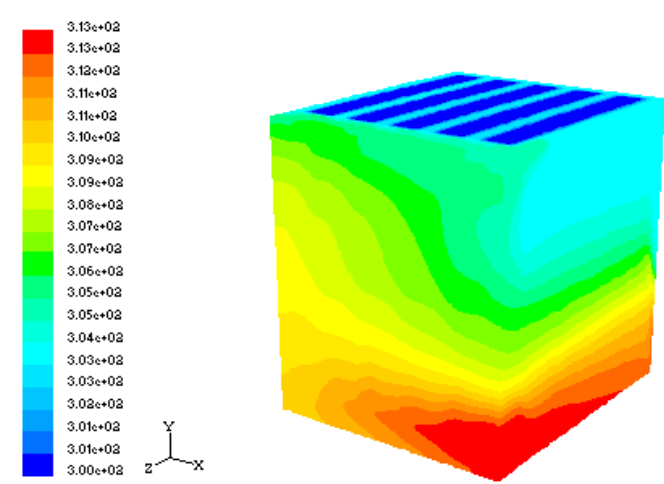

Fig.10 Temperature distributing of the experimental model

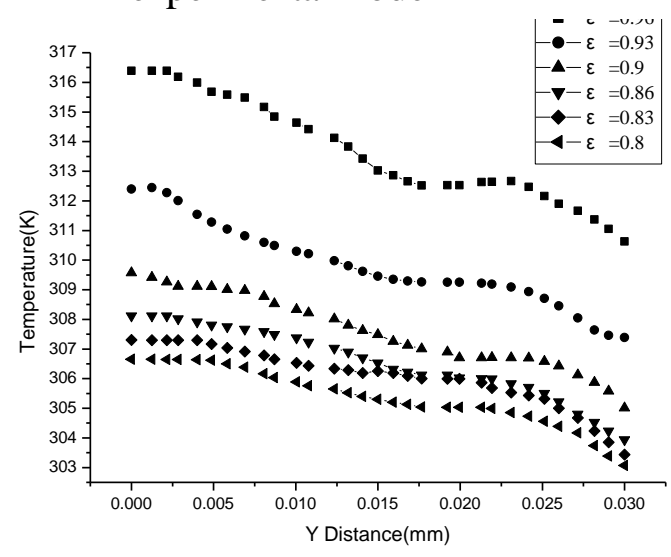

Fig. 12 Curves of temperature varying with porosity

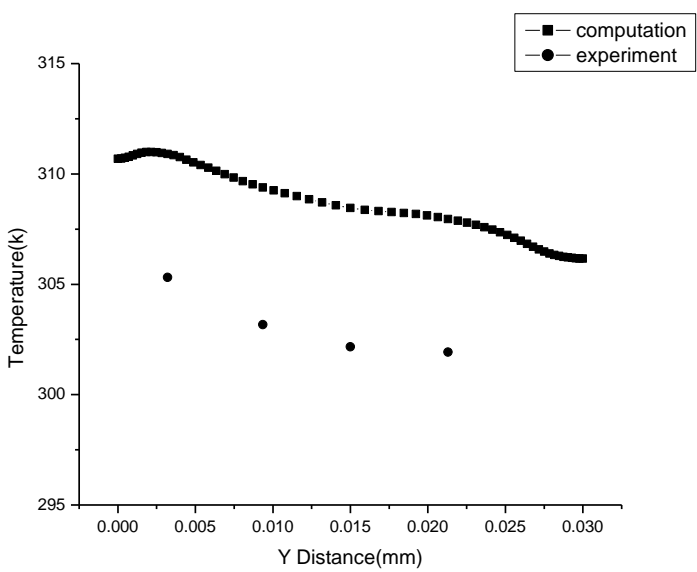

Fig. 11The comparison of numerical with experimental result

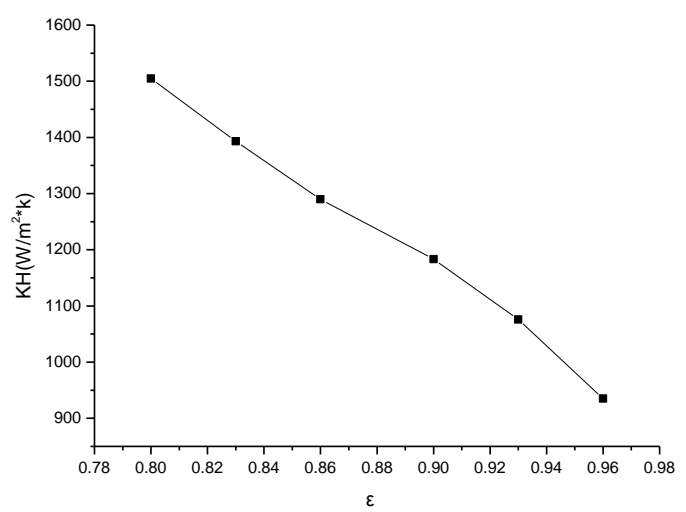

Fig.13 Curves of KH varying with porosity

The research [9] indicates that porous medium can strengthen heat transfer, but the flow drag increases more when the porosity is less than 0.8 . So the design of the new cooling heat transfer configuration takes big porosity porous medium into account. It can strengthen heat transfer, but the flow drag does not increase much. Therefore, the paper adopts big porosity porous medium in numerical simulation $(\varepsilon \geqslant 80 \%)$. Fig 12 and Fig13 illustrate the diagrams that the temperature on the heating surface and the parameters of thermo-driving heat transfer capacity change along with porosity under the conditions $\left(\mathrm{q}=5390 \mathrm{~W} / \mathrm{m}^{2}, \mathrm{n}=900 \mathrm{rpm}\right.$, $\mathrm{v}=37.4 \mathrm{~m} / \mathrm{s})$. Fig 12 illustrates heating surface temperature and the temperature difference between the highest and lowest measuring points decreases along with the decrease of porosity. That demonstrates porosity decreases, thermo-driving heat transfer capacity rises. Fig13 also proves that. Fig12 also illustrates the decline extent of heating surface temperature and the temperature difference between the highest and lowest measuring points decreases along with the decrease of porosity. Fig13 illustrates the increase extent of the 
parameter of thermo-driving heat transfer capacity decreases along with the decrease of porosity. These analyses demonstrate thermo-driving heat transfer capacity increases but its increase extent decreases along with the decrease of porosity. The reason is as follows: when the porosity decreases, the addition of contact area of solid medium and fluid strengthens convection heat transfer, but the increase of flow drag weakens convection heat transfer. It leads to the decrease of increase extent of thermo-driving heat transfer capacity finally.

\section{Conclusion}

(1) The flow of thermo-driving takes place in the new cooling configuration after being filled with porous medium.

(2) The new cooling configuration has the same heat transfer rule under different porosity: when rotating speed, heat flux and intake velocity of the cooling air increases, its thermo-driving heat transfer capacity also rises.

(3) The new cooling configuration's thermo-driving heat transfer capacity increases but its increase extent decreases along with the decrease of porosity.

\section{References}

[1] Chang H, Guo Z. A new cooling technique used in turbine blade. Invention patent No. ZL01108222, 2001-04.

[2] M.Varahasamy,R.M.Fand.Heat Transfer by Forced Convection in Pipes Packed with Media Whose Matrices Are Composed of Spheres.Int. [J].Heat Mass Transfer,1996, 39(18): 3931 3847.

[3] Hsu.C.T.,Cheng P.. Closure Scheme of the Macroscopic Energy Equation for Convection Heat Transfer in Porous Media.Int. [J].Commu.Heat Transfer, 1988, 15:689 703

[4] Morrison H L et al.[J]. Appl. Phys., 1949,20:1027 1029

[5] Mao Junkui, Chang Haiping, Fang Li. Experimentallnvestigation of Flow and Heat Transfer About the Thermal Driving of R12 in Rotating Small-Size CircularEnclosures[J].Journal of Aerospace Power,2003,18(4): 498 501.

[6] Mao Junkui, Chang Haiping, Fang Li. Investigation of Thermally Driven in Centrifugal Field with Liquid Crystal [J]. Journal of Engineering Thermophysics,2004,25(2): 229 231.

[7]Yang Min, Chang Haiping, Mao Junkui. Experimentallnvestigation of Thermally Driving Heat TransferCharacteristics of $\mathrm{H} 2 \mathrm{O}$ and R12 in a Centrifugal Force Field [J].Journal of Engineering Thermophysics,2004,25(2): 302 304.

[8] Fluent User's Guide, 1998

[9] Renken K.J,Poulikakous D. Experiment and Analysis of Forced Convection Heat Transport in a Packed Bed of Sphere, Int [J].Heat Mass Transfer 1998,31: 1399 1408 